CLINICAL STUDY

\title{
Decreased ovarian function is associated with obesity in very long-term female survivors of childhood cancer
}

\author{
W van Dorp ${ }^{1,2, *}$, K Blijdorp ${ }^{1,3, *}$, J S E Laven ${ }^{2}$, R Pieters ${ }^{1}$, J A Visser ${ }^{4}$, A J van der Lely ${ }^{3}$, S J C M M Neggers ${ }^{1,3}$ \\ and $\mathrm{M}$ M van den Heuvel-Eibrink ${ }^{1}$ \\ ${ }^{1}$ Department of Paediatric Oncology/Haematology, Erasmus MC-Sophia Children's Hospital, Dr Molewaterplein 60, 3015 GJ, Rotterdam, \\ The Netherlands, ${ }^{2}$ Department of Gynaecology and Obstetrics, Subdivision Reproductive Medicine, ${ }^{3}$ Department of Medicine - section Endocrinology and \\ ${ }^{4}$ Department of Internal Medicine, Erasmus MC-University Medical Center Rotterdam, s' Gravendijkwal 230, 3015 CE, Rotterdam, The Netherlands \\ (Correspondence should be addressed to $W$ van Dorp who is now at Department of Gynaecology and Obstetrics, and Paediatric Oncology/Haematology, \\ Erasmus MC-University Medical Center, PO Box 2040, 3000 CA Rotterdam, The Netherlands; Email: w.vandorp@erasmusmc.nl)
}

*(W van Dorp and K Blijdorp shared first authorship).

\begin{abstract}
Objective: Obesity and gonadal dysfunction are known major side effects of treatment in adult childhood cancer survivors (CCS). In the general population, obesity has a negative influence on female fertility. We aimed to evaluate whether obesity and serum insulin are associated with decreased ovarian reserve markers in CCS.

Design: Retrospective single-center cohort study.

Methods: Data of 191 female survivors of childhood cancer were analyzed. Median follow-up time was 18.8 (2.3-48.8) years. Outcome measures were serum anti-Müllerian hormone (AMH) and total follicle count (FC). Potential risk factors were: BMI; body composition measures, determined by dualenergy X-ray absorptiometry (total fat percentage, lean body mass, and visceral fat percentage); and fasting insulin.

Results: Lower serum AMH was found in obese subjects $(\beta(\%)-49, P=0.007)$ and in subjects with fasting insulin in the highest tertile $(\beta(\%)-43, P=0.039)$. Total fat percentage tends to be associated with serum AMH $(\beta(\%)-2.1, P=0.06)$. Survivors in the highest tertile of insulin had significantly lower FC than survivors in the lowest tertile $(\beta-6.3, P=0.013)$. BMI and other measures of body composition were not associated with FC. Correlation between serum AMH and antral follicle count (AFC) was $\rho=0.32(P=0.08)$.

Conclusions: Obesity and insulin resistance are associated with gonadal damage, as reflected by decreased $\mathrm{AMH}$ and reduced FC in adult survivors of childhood cancer. In contrast to its highly predictive value for AFC in the healthy female population, serum AMH does not seem to correlate as well with AFC in CCS.
\end{abstract}

European Journal of Endocrinology $168905-912$

\section{Introduction}

The prevalence of obesity has increased dramatically since the 1970s (1). It is a well-described risk factor for diabetes, hypertension, heart disease, stroke, and cancer (2). In healthy women, high BMI also affects reproductive health $(3,4)$ as reflected by impaired ovulatory function and a lower pregnancy rate (5). In childhood cancer survivors (CCS), obesity is a major side effect, occurring in $9-30 \%$, and mainly depending on previous treatment $(6,7)$. In adult female CCS, the risk of obesity is increased by $50 \%$ when compared with the general population (8).

Gonadal dysfunction is an important side effect of cancer treatment in CCS. Alkylating agents and abdominal radiotherapy, in particular, can have a deleterious effect on ovarian function (9). To determine ovarian reserve, anti-Müllerian hormone (AMH) was identified as a reliable serum marker (10). It is produced by the granulosa cells of small growing follicles, reflects the size of the primordial follicle pool in the ovaries, and is an indicator of a woman's reproductive capacity (11). It is stable during and between menstrual cycles, in contrast to FSH $(12,13,14)$, and is considered to be a valued marker for ovarian reserve, as it corresponds well with antral follicle count (AFC), which reflects reproductive status (10).

In their late reproductive years, the gonadal function of women seems to be affected by obesity $(15,16)$. Insulin resistance, which occurs at a higher frequency 
in adult CCS, may affect granulosa cell function $(17,18)$. The association between obesity, insulin resistance, and gonadal function, which are more prevalent in CCS than in the normal population, has never been explored. Therefore, the aim of this study was to determine the association between BMI, body composition, insulin levels, and ovarian reserve as reflected by AMH and AFC in a substantial single center cohort of female adult survivors of childhood cancer.

\section{Materials and methods}

\section{Subjects}

A retrospective single-center cohort study was performed among female survivors that visited our lateeffects outpatient clinic for long-term CCS. Inclusion criteria were age $\geq 18$ and $<50$ years and female CCS diagnosed and treated between 1964 and 2005, who had both serum AMH levels and BMI determined at the same moment at least 5 years after cessation of cancer treatment. Exclusion criteria were ovariectomy, polycystic ovary syndrome (PCOS), and AMH $>5 \mu \mathrm{g} / \mathrm{l}$ (Supplementary Figure 1, see section on supplementary data given at the end of this article). One hundred and five survivors visited the gynecological outpatient clinic for screening. In the remaining 180 CCS, we were not able to distinguish between PCOS and nonPCOS subjects because data on hyperandrogenism (clinical and biochemical) and total follicle counts (FC; transvaginal ultrasound) were not available. As we were not able to use the Rotterdam criteria in these remaining cases, and Dewailly et al. (19) suggested a cutoff limit of AMH $>5 \mu \mathrm{g} / \mathrm{l}$ to define PCOM, we used this marker and cutoff limit for the presence of PCOM as one of the criteria of PCOS and excluded these survivors.

\section{Outcome measures (AMH/FC)}

Serum samples were taken randomly during the menstrual cycle as AMH has been shown not to fluctuate during the menstrual cycle or during OCP use (12). AMH was measured using an in-house double-antibody ELISA (commercially available through Beckman-Coulter) $(10,20)$. The intra- and interassay coefficients of variation were $<10$ and $<5 \%$ respectively $(10,20)$. A subset of patients $(n=91)$ underwent a standardized gynecological examination. Clinical examination was performed after an overnight fasting period on a random day and included menstrual history, current cycle length, cycle regularity, height, and weight. Transvaginal ultrasonography was performed to assess ovarian volume and total FC for both ovaries and to exclude other genital abnormalities. FC was called AFC if the FC was performed during the follicular phase (days 2-5) or during amenorrhea. PCOS was diagnosed according to the revised Rotterdam 2003 criteria (21). The presence of polycystic ovaries was defined as $\geq 12$ follicles in one or both ovaries and/or increased ovarian volume $(>10 \mathrm{ml})$, without the presence of a cyst $(>10 \mathrm{~mm})(22)$.

\section{Obesity variables}

Follow-up data of the most recent visit included the following variables: weight, height, BMI calculated from height and weight (23), and waist:hip ratio (WHR), as measured by waist circumference divided by hip circumference (24). Serum insulin (pmol/l) was measured using a chemiluminescence-based immunoassay (Immulite 2000, Siemens DPC, Los Angeles, CA, USA) after an overnight fasting period. Glucose levels were measured using a Hitachi 917 analyzer (Roche Diagnostics). Insulin resistance was determined by calculation of the homeostasis model assessment (HOMA) score as plasma glucose $(\mathrm{mmol} / \mathrm{l}) \times$ plasma insulin (mU/l)/22.5 (25). Lean body mass $(\mathrm{kg})$ and percentage of body fat were measured by dual-energy X-ray absorptiometry (DXA, Lunar Prodigy, GE Healthcare, Madison, WI, USA). Visceral fat percentage was calculated from intra-abdominal fat $(\mathrm{kg})$ and total fat (kg) measured by DXA (26). Waist, WHR, and visceral fat percentage were not analyzed in the subset of survivors treated with abdominal radiotherapy because of impairment of local body fat and the frequent occurrence of scoliosis.

\section{Potential confounders}

Data concerning treatment protocols, disease, and patient characteristics were retrieved from our local database and were completed using the medical records where necessary. Follow-up time was defined as the time since cessation of treatment. Among patients exposed to alkylating agents, the alkylating agent dose (AAD) score was calculated by determining the drug dose tertile distribution in our entire cohort of survivors and adding the tertile scores $(1,2$, and 3$)$ for each of the alkylating agents given to a particular patient as described previously by Green et al. and Tucker et al. $(27,28)$. An AAD score of zero was assigned to patients not exposed to any alkylating agent.

\section{Statistical analysis}

To examine the associations between obesity variables and $\mathrm{AMH}$ or $\mathrm{FC}$ we used univariate and multiple linear regression analyses. In all multiple linear regression models, age, age at diagnosis, total body irradiation, abdominal radiotherapy, and AAD score were included as potential confounders. The analyses were performed in several steps. First, BMI, body composition, and insulin were entered as continuous variables. Secondly, to evaluate whether there was an exponential 
association with $\mathrm{AMH}$, squared variables of BMI, body composition measures, and insulin were added to the relevant model. Subsequently, all variables were divided into quintiles or tertiles (depending on sample size) and added to the relevant model as dummy variables, with the lowest quintile/tertile as reference category. Additionally, BMI was divided into four categories: $\geq 30 \mathrm{~kg} / \mathrm{m}^{2}$ (obese), 25-30 kg/m² (overweight), $18-25 \mathrm{~kg} / \mathrm{m}^{2}$ (normal weight), and $<18 \mathrm{~kg} / \mathrm{m}^{2}$ (underweight) and added to the model as dummy variables with normal weight as reference category. Associations are expressed as standardized regression coefficients because this measure allows direct comparison of the strengths of associations between different determinants. The distribution of $\mathrm{AMH}$ was normalized by

${ }^{10} \log$ transformation to improve the plots of the residual analyses and expressed as percentage. $P$ values $<0.05$ (two-tailed) are considered statistically significant. SPSS 17.0 software (SPSS) was used for statistical analysis.

\section{Results}

\section{Gonadal function}

The cohort consisted of 425 female survivors of childhood cancer, of whom 292 visited the late-effects outpatient clinic. Seven survivors were excluded because of previous one-sided or two-sided ovariectomy ( $n=6$ and $n=1$ respectively) and two because they were $>50$ years old. Sixteen survivors were clinically diagnosed with PCOS by a gynecologist and were therefore excluded. Another 76 survivors were excluded because their AMH levels were $>5 \mu \mathrm{g} / \mathrm{l}$. Finally, 191 female survivors were included in the analysis.

Clinical characteristics and treatment details of the total cohort of female CCS of our center and the survivors included in the study are shown in Table 1. The included sample is representative for the total cohort of female CCS of our center.

At the time of inclusion, 42 of 191 included survivors $(22.0 \%)$ had regular menstrual cycles, whereas eight survivors (4.2\%) had an oligo- or amenorrhea. One survivor had shortening of mean menstrual cycle length (1.3\%). No data on menstrual cycles were available in one survivor (1.3\%) who delivered recently and in one survivor $(1.3 \%)$ who was pregnant. In 23 of 191 survivors (30.3\%), data on menstrual cycle at the time of screening were not available. All other survivors used oral contraceptive pills $(99 / 191,51.8 \%)$ or were under hormonal replacement therapy (16/191: $8.4 \%)$ at the time of follow-up.

Median AMH level of the total group was 1.7 (range 0.1-4.9) $\mu \mathrm{g} / \mathrm{l}$. Median total FC was $10(0-25)$.

Table 1 Representativeness of included survivors compared with the total group of female adult CCS and survivors in whom AMH was measured.

\begin{tabular}{|c|c|c|c|c|}
\hline & & $\begin{array}{l}\text { Total group of } \\
\text { adult female } \\
\text { CCS }(n=425)\end{array}$ & $\begin{array}{l}\text { Female CCS with } \\
\text { AMH measurement } \\
(n=292)\end{array}$ & $\begin{array}{l}\text { Included survivors } \\
\text { in this study } \\
\qquad(n=191)\end{array}$ \\
\hline Age at diagnosis (years) & & $7.3(0-17.9)$ & $6.2(0-16.8)$ & $6.3(0-16.2)$ \\
\hline Age at follow-up (years) & & NA & $26.5(17.7-57.4)$ & $27.1(17.7-50.0)$ \\
\hline Follow-up time (years) & & NA & $17.8(2.3-48.8)$ & $18.8(2.3-48.8)$ \\
\hline \multicolumn{5}{|l|}{ Diagnosis $n(\%)$} \\
\hline ALL and T-NHL & & $128(30)$ & $91(31)$ & $52(27)$ \\
\hline Acute myeloid leukemia & & $9(2)$ & $9(3)$ & $7(4)$ \\
\hline B-cell non-Hodgkin lymphoma & & $25(6)$ & $18(6)$ & $12(6)$ \\
\hline Hodgkin lymphoma & & $29(7)$ & $21(7)$ & $18(9)$ \\
\hline Bone tumor & & $22(5)$ & $14(5)$ & $14(7)$ \\
\hline Wilms tumor & & 47 (11) & 41 (14) & $31(16)$ \\
\hline Neuroblastoma & & $32(8)$ & $29(10)$ & $18(9)$ \\
\hline Germ cell tumor & & $12(3)$ & $8(3)$ & $2(1)$ \\
\hline Malignant mesenchymal tumor & & $35(8)$ & $27(9)$ & $17(9)$ \\
\hline Brain tumor & & $52(12)$ & $18(6)$ & $13(7)$ \\
\hline Other & & $34(8)$ & $16(5)$ & $7(4)$ \\
\hline Therapy $n(\%)$ & $n(\%)$ & TCD (Gy) & TCD (Gy) & TCD (Gy) \\
\hline Abdominal radiotherapy & $27(7)$ & $25(10-71)$ & $25(10-62)$ & $25(10-60)$ \\
\hline \multicolumn{4}{|l|}{ AAD score } & $12(7-12)$ \\
\hline 0 & & $237(56)$ & $150(51)$ & $93(49)$ \\
\hline 1 & & $47(11)$ & $35(12)$ & $19(10)$ \\
\hline 2 & & $47(11)$ & $39(13)$ & $26(14)$ \\
\hline 3 & & $70(17)$ & 55 (19) & $42(22)$ \\
\hline 4 & & $9(2)$ & $5(2)$ & $5(3)$ \\
\hline$\geq 5$ & & $15(4)$ & $8(2)$ & $6(3)$ \\
\hline
\end{tabular}

NA, not applicable; CCS, childhood cancer survivors; AMH, anti-Müllerian hormone; ALL, acute lymphoblastic leukemia; T-NHL, T-cell non-Hodgkin lymphoma; TCD, total cumulative dose; $A A D$, alkylating agent dose.

${ }^{a}$ After exclusion of ovariectomized subjects $(n=7)$, PCOS subjects as classified according to the revised PCOS Rotterdam criteria $(n=16)$, AMH $>5 \mu \mathrm{g} / \mathrm{l}$ $n=76$ ) if subjects were not classified according to the revised PCOS Rotterdam criteria since information about follicle count and hyperandrogenism was not available, and women $>50$ years $(n=2)$. Data are expressed as median (range) or frequencies $(\%)$. 
Table 2 Univariate and multivariate linear regression analyses illustrating the influence of BMI, measures of body composition, and insulin on AMH levels.

\begin{tabular}{|c|c|c|c|c|c|c|}
\hline & \multicolumn{6}{|c|}{ AMH $(\%) n=191(122)^{a}$} \\
\hline & \multicolumn{3}{|c|}{ Univariate model } & \multicolumn{3}{|c|}{ Multivariate model } \\
\hline & $\beta(\%)$ & $95 \% \mathrm{Cl}$ & $P$ value & $\beta(\%)$ & $95 \% \mathrm{Cl}$ & $P$ value \\
\hline Obesity $\left(\mathrm{BMI} \geq 30 \mathrm{~kg} / \mathrm{m}^{2}\right)$ & $-55^{\star}$ & $-75 ;-18$ & 0.009 & $-49^{\star}$ & $-68 ;-17$ & 0.007 \\
\hline Overweight (BMI $\left.25-30 \mathrm{~kg} / \mathrm{m}^{2}\right)$ & 1 & $-35 ; 56$ & 0.974 & 15 & $-20 ; 64$ & 0.450 \\
\hline Underweight $\left(\mathrm{BMI}<18 \mathrm{~kg} / \mathrm{m}^{2}\right)$ & -17 & $-68 ; 116$ & 0.698 & -21 & $-64 ; 76$ & 0.568 \\
\hline BMI & -3.3 & $-6.8 ; 0.4$ & 0.081 & -2.0 & $-5.0 ; 1.0$ & 0.191 \\
\hline Total fat percentage & $-3.7^{*}$ & $-6.4 ;-1.0$ & 0.009 & -2.1 & $-4.2 ; 0.1$ & 0.06 \\
\hline Lean body mass $(\mathrm{kg})$ & 2.0 & $-11.3 ; 5.2$ & 0.22 & 0.4 & $-2.2 ; 3.0$ & 0.76 \\
\hline Waist circumference $(\mathrm{cm})^{\mathrm{b}}$ & $-2.0^{\star}$ & $-3.7 ;-0.2$ & 0.025 & -0.5 & $-1.8 ; 0.9$ & 0.511 \\
\hline Waist:hip ratio ${ }^{\mathrm{b}}$ & $-2.5^{*}$ & $-4.3 ;-0.2$ & 0.037 & 0.1 & $-1.8 ; 2.6$ & 0.894 \\
\hline Visceral fat percentage $^{b}$ & $-13^{*}$ & $-22 ;-2$ & 0.02 & -1 & $-10 ; 8$ & 0.79 \\
\hline Insulin & -0.4 & $-0.9 ; 0.1$ & 0.132 & -0.2 & $-0.6 ; 0.3$ & 0.48 \\
\hline Insulin 2nd tertile (24-54 pmol//) & 2 & $-47 ; 96$ & 0.952 & -8 & $-45 ; 55$ & 0.761 \\
\hline Insulin 3rd tertile (> $>54 \mathrm{pmol} / \mathrm{l})$ & $-54^{*}$ & $-76 ;-12$ & 0.019 & $-43^{*}$ & $-67 ;-3$ & 0.039 \\
\hline HOMA & -13 & $-28 ; 5$ & 0.135 & -6 & $-18 ; 9$ & 0.387 \\
\hline HOMA 2nd tertile $(0.58-1.40)$ & 17 & $-40 ; 127$ & 0.636 & -8 & $-46 ; 9$ & 0.753 \\
\hline HOMA 3rd tertile $(>1.40)$ & -45 & $-71 ; 8$ & 0.080 & -41 & $-65 ; 0.3$ & 0.051 \\
\hline
\end{tabular}

$\mathrm{AMH}$, anti-Müllerian hormone; HOMA, homeostasis model assessment. ${ }^{*} P<0.05$

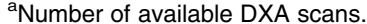

${ }^{b}$ For the dependent variables, waist, waist:hip ratio, and visceral fat percentage, survivors treated with abdominal radiotherapy are excluded from the analysis $(n=19)$. Corrected for age, age at diagnosis, total body irradiation, abdominal radiotherapy, and alkylating agent dose (AAD) score.

The Spearman correlation coefficient $(\rho)$ between AMH and AFC was $0.32(P=0.08)$ in survivors who were screened during the follicular phase or during amenorrhea. FSH was significantly inversely correlated with AMH $(\rho=-0.30, P<0.001)$ and was significantly higher in survivors with AMH $<0.1 \mu \mathrm{g} / \mathrm{l}$ compared with survivors with AMH $>0.1 \mu \mathrm{g} / \mathrm{l}(20.9$ vs $4.9 \mathrm{U} / \mathrm{l})$.

\section{Influence of obesity, body composition, and insulin on AMH}

Twenty subjects (10\%) were defined as obese (BMI $\left.\geq 30 \mathrm{~kg} / \mathrm{m}^{2}\right), 25(13 \%)$ as overweight (BMI $25-30 \mathrm{~kg} / \mathrm{m}^{2}$ ), seven $(4 \%)$ as underweight (BMI $<18 \mathrm{~kg} / \mathrm{m}^{2}$ ), and $121(63 \%)$ had normal weight (Supplementary Table 1 , see section on supplementary data given at the end of this article). AMH levels were significantly inversely associated with obesity (BMI $\geq 30 \mathrm{~kg} / \mathrm{m}^{2}$ ), high fasting insulin $(>54 \mathrm{pmol} / \mathrm{l})$, total fat percentage, waist circumference, WHR, and visceral fat percentage but not with HOMA and lean body mass (Table 2). After adjustment for confounders (age, age at diagnosis, treatment with abdominal or total body irradiation, and AAD score) obesity (BMI $>30 \mathrm{~kg} / \mathrm{m}^{2}$ ) and high fasting insulin $(>54 \mathrm{pmol} / \mathrm{l})$ remained significantly associated with AMH $(\beta(\%)-48, P=0.008$, and $\beta(\%)-43$, $P=0.039$, respectively) (Table 2 and Figs 1 and $2 \mathrm{a}$ and $\mathrm{b}$ ).

\section{Influence of obesity, body composition, and insulin on FC}

There were no significant associations between FC and BMI or body composition measures (Table 3). Survivors with insulin and HOMA in the highest tertile had significantly lower FC than others. After adjustment for confounders, no linear or exponential association between FC and BMI or measures of body composition was found. FC did not differ significantly between quintiles of BMI or body composition (data not shown). Subsequently, FC did not differ between BMI categories. However, there were only five obese subjects with available FCs (data not shown). Insulin was

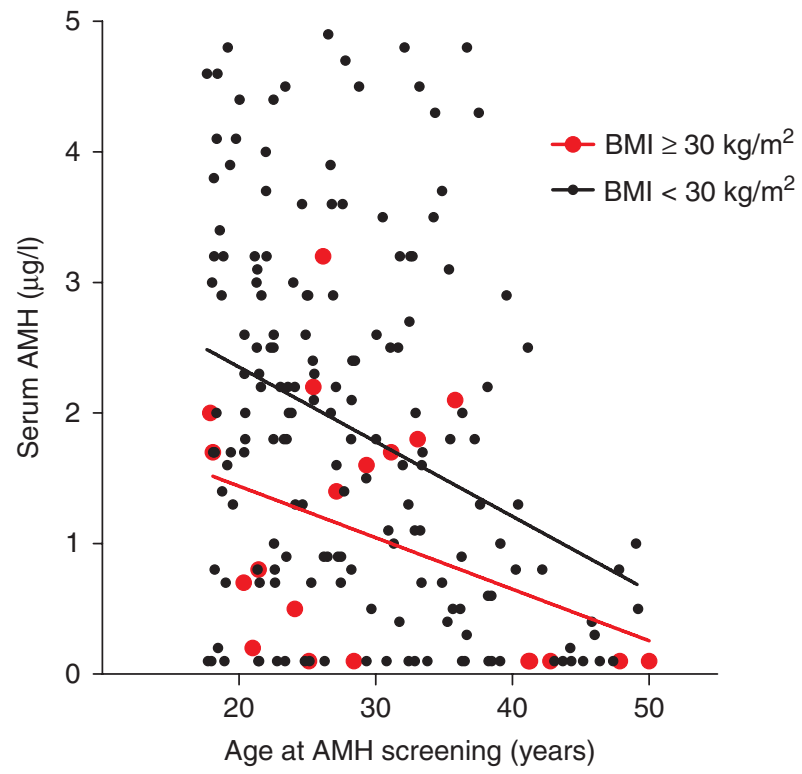

Figure 1 Anti-Müllerian hormone $(\mathrm{AMH})$ in obese $\left(\mathrm{BMI} \geq 30 \mathrm{~kg} / \mathrm{m}^{2}\right)$ survivors compared with nonobese survivors. 
significantly associated with FC, i.e. survivors with an insulin level in the highest tertile $(>48 \mathrm{pmol} / \mathrm{l})$ had significantly lower FC counts when compared with survivors with insulin levels $<25 \mathrm{pmol} / \mathrm{l}(\beta-6.3$, $P=0.013$ ) (Table 3 and Fig. 2c).
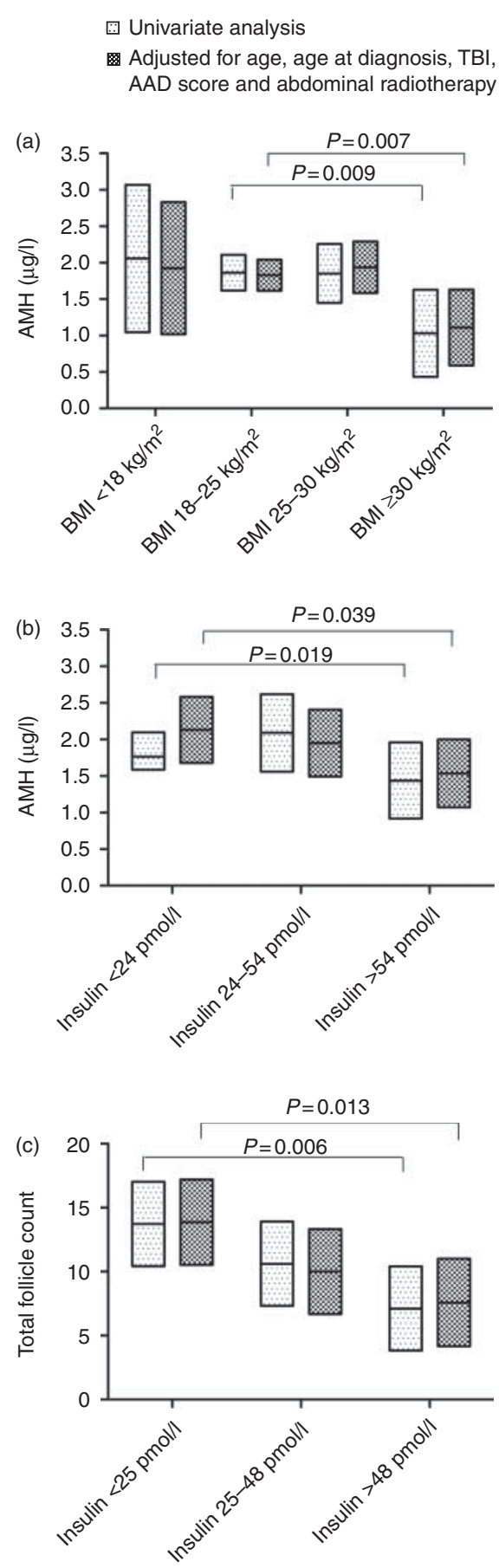

Figure 2 (a) Anti-Müllerian hormone (AMH) in four BMI categories, univariate and after adjustment for possible confounders expressed as mean $(95 \% \mathrm{Cl})$. (b) $\mathrm{AMH}$ in serum fasting insulin subgroups expressed as mean $(95 \% \mathrm{Cl})$. (c) Total antral follicle count in serum fasting insulin subgroups expressed as mean $(95 \% \mathrm{Cl})$.

\section{Discussion}

The current study shows that diminished ovarian reserve, as reflected by low $\mathrm{AMH}$ and low $\mathrm{FC}$, was independently associated with obesity and high insulin levels in female CCS. Our results show that obesity is indeed independently associated with decreased AMH in female CCS $(6,29,30)$. In the current study, total and visceral fat percentages were not associated with gonadal function, although we observed a trend to a negative association between total fat percentage and AMH levels. This may be due to a size effect or to the fact that we measured total fat percentage and not the fat distribution in different compartments. In fact, for that purpose, abdominal computed tomography, which is the gold standard for measuring intra-abdominal fat mass, is preferred over DXA (31).

Although no oral glucose tolerance tests were performed and therefore some cases in the prediabetic state might have been missed, none of the subjects were diagnosed and treated for diabetes mellitus, based on fasting glucose levels and/or medical history. Therefore, we evaluated fasting insulin levels as a measure for insulin resistance and related these to ovarian reserve markers. The negative association between fasting insulin levels and AMH was previously described in the reproductive-age women without PCOS (16) and was confirmed by our study among CCS.

We identified obesity to be negatively associated with ovarian reserve as assessed by AMH levels. There is no linear association between AMH and BMI, but above a certain threshold (in this case, BMI $30 \mathrm{~kg} / \mathrm{m}^{2}$ ), AMH is significantly lower compared with normal weight subjects. The explanation for this nonlinear association could be that only above a certain threshold of BMI do metabolic changes occur, including insulin resistance, leptin resistance, and elevated levels of adipokines. These factors could play a role in affecting the pituitarygonadal axis and damaging granulosa cells, although this hypothesis has never been proved. In this study, all subjects with obesity were evaluated, including one survivor treated for craniopharyngioma and one survivor treated with high-dose brain tumor irradiation (>35 Gy). In these subjects, hypothalamic obesity could not be excluded. To our knowledge, no other studies that assess a possible link between obesity and AMH have been performed in female CCS. In the general population, only one study among three has shown an association in women of reproductive age $(15,16,32)$.

Based on these results, we hypothesize that obesity influences the degree of gonadal damage in female CCS. However, it is also conceivable that impaired gonadal function may lead to the development of adiposity and insulin resistance. In animal models, it is known that estradiol has an inhibitory effect on food intake via anorexigenic peptides that decrease meal size. In ovariectomized rats, the removal of estrogens leads to changes in meal size, obesity $(33,34)$, increased leptin 
Table 3 Multivariate linear regression analyses illustrating the lacking influence of BMI, measures of body composition, and insulin on total follicle count.

\begin{tabular}{|c|c|c|c|c|c|c|}
\hline & \multicolumn{6}{|c|}{ Total follicle count $n=54(34)^{a}$} \\
\hline & \multicolumn{3}{|c|}{ Univariate model } & \multicolumn{3}{|c|}{ Multivariate model } \\
\hline & $\beta(\%)$ & $95 \% \mathrm{Cl}$ & $P$ value & $\beta(\%)$ & $95 \% \mathrm{Cl}$ & $P$ value \\
\hline $\begin{array}{l}\left.\text { Obesity (BMI } \geq 30 \mathrm{~kg} / \mathrm{m}^{2}\right) \\
\text { Overweight }\left(\mathrm{BMI} 25-30 \mathrm{~kg} / \mathrm{m}^{2}\right) \\
\text { Underweight }\left(\mathrm{BMI}<18 \mathrm{~kg} / \mathrm{m}^{2}\right) \\
\text { BMI } \\
\text { Total fat percentage } \\
\text { Lean body mass }(\mathrm{kg}) \\
\text { Waist circumference }(\mathrm{cm})^{\mathrm{b}} \\
\text { Waist:hip ratio } \\
\text { Visceral fat percentage } \\
\text { Insulin } \\
\text { Insulin 2nd tertile }(25-48 \mathrm{pmol} / \mathrm{l}) \\
\text { Insulin 3rd tertile }(>48 \mathrm{pmol} / \mathrm{l}) \\
\text { HOMA } \\
\text { HOMA 2nd tertile }(0.66-1.24) \\
\text { HOMA 3rd tertile }(>1.24)\end{array}$ & $\begin{array}{l}-0.6 \\
-2.1 \\
-3.0 \\
-0.03 \\
-0.09 \\
0.18 \\
-0.02 \\
-0.06 \\
-0.58 \\
-0.03 \\
-3.1 \\
-6.6^{\star} \\
-0.98 \\
-2.47 \\
-5.06^{\star}\end{array}$ & $\begin{array}{l}-7.8 ; 6.6 \\
-7.7 ; 3.5 \\
-13.9 ; 7.9 \\
-0.43 ; 0.38 \\
-0.44 ; 0.26 \\
-0.15 ; 0.51 \\
-0.24 ; 0.21 \\
-0.37 ; 0.25 \\
-2.44 ; 1.28 \\
-0.07 ; 0.00 \\
-7.8 ; 1.5 \\
-11.3 ;-2.0 \\
-2.24 ; 0.27 \\
-7.22 ; 2.28 \\
-9.95 ;-0.18\end{array}$ & $\begin{array}{l}0.867 \\
0.452 \\
0.584 \\
0.902 \\
0.614 \\
0.266 \\
0.890 \\
0.696 \\
0.529 \\
0.083 \\
0.186 \\
0.006 \\
0.121 \\
0.302 \\
0.043\end{array}$ & $\begin{array}{l}-0.9 \\
-2.1 \\
-5.4 \\
-0.01 \\
-0.06 \\
0.20 \\
0.04 \\
0.05 \\
-0.18 \\
-0.02 \\
-3.9 \\
-6.3^{*} \\
-0.70 \\
-3.42 \\
-5.01\end{array}$ & $\begin{array}{l}-8.0 ; 6.2 \\
-8.1 ; 3.8 \\
-16.4 ; 5.6 \\
-0.42 ; 0.40 \\
-0.44 ; 0.32 \\
-0.17 ; 0.57 \\
-0.18 ; 0.26 \\
-0.26 ; 0.35 \\
-2.10 ; 1.75 \\
-0.06 ; 0.01 \\
-8.6 ; 0.8 \\
-11.2 ;-1.4 \\
-2.00 ; 6.00 \\
-8.40 ; 1.57 \\
-10.26 ; 0.23\end{array}$ & $\begin{array}{l}0.804 \\
0.471 \\
0.330 \\
0.978 \\
0.747 \\
0.278 \\
0.732 \\
0.759 \\
0.851 \\
0.192 \\
0.104 \\
0.013 \\
0.283 \\
0.175 \\
0.061\end{array}$ \\
\hline
\end{tabular}

HOMA, homeostasis model assessment. ${ }^{*} P<0.05$

${ }^{a}$ Number of available DXA scans.

${ }^{b}$ For the dependent variables, waist, waist:hip ratio, and visceral fat percentage, survivors treated with abdominal radiotherapy are excluded from the analysis $(n=19)$. Corrected for age, age at diagnosis, total body irradiation, abdominal radiotherapy, and AAD score.

sensitivity, and decreased insulin sensitivity (35). However, insulin resistance could also decrease granulosa cell function, which could lead to reduced ovarian function and therefore lower AMH levels $(17,18)$. Our hypothesis fits with the result of a study in type 2 diabetes mellitus (T2DM) patients, in which AMH levels were significantly lower than in healthy controls (36), which was possibly a result of insulin resistance in the T2DM patients. Furthermore, the fact that stringent glycemic control in diabetic patients improves menstrual cycles and fertility rates underlines the hypothesis that prolonged hyperglycemia and chronic complications of diabetes negatively affect ovarian reserve (37). Animal models have shown that ovulation was suppressed in hyperglycemic-hyperinsulinemic conditions, due to hypovascularization, follicular atresia, and eventually involution of ovaries, caused by glucotoxicity and the cytolipotoxic effect of obesity (38). It should, however, be stressed that due to the cross-sectional design of the current study, cause and effect could not be distinguished.

In 105/285 cases, PCOS diagnosis was verified to confirm the Rotterdam criteria, as these survivors also visited the gynecological outpatient clinic. However, in the remaining 180 CCS, we were not able to distinguish between PCOS and non-PCOS subjects because data on hyperandrogenism (clinical and biochemical) and total FC (transvaginal ultrasound) were not available. As we were not able to use the Rotterdam criteria in these remaining cases, and Dewailly et al. (19) suggested a cutoff limit of AMH $>5 \mu \mathrm{g} / \mathrm{l}$ to define PCOM, we used this marker and cutoff limit for the presence of PCOM.
We recognize the limitation of this cutoff limit as we probably excluded more subjects than we would have done if we were able to exclude them based on the Rotterdam criteria. However, we believe that this is the best way to make our population as homogeneous as possible. Moreover, the remaining subjects were representative of the whole cohort of female CCS according to age, age at follow-up, diagnosis, and treatment. As we agreed that the use of the cutoff value is of limited accuracy, we also performed sub-analyses in the 105 cases that were classified based on the Rotterdam criteria. Sixteen survivors were diagnosed with PCOS and were therefore excluded from this analysis. We found a trend to an association between total fat percentage and AMH, although not significant $(P=0.053)$. This is the same trend as found in our previous analyses. However, we did not find an association between BMI and AMH, which might be due to the underrepresentation of obese survivors in this subset $(n=5)$. If we perform multivariate analyses in the whole group of survivors with AMH levels $(n=285)$, we observe no significant correlations with obesity, which fits with the hypothesis that obese women with PCOS have raised AMH levels, while obese women without PCOS have lower AMH levels.

Despite the negative association between $\mathrm{FC}$ and serum insulin, we did not find an association between FC and obesity, in contrast to AMH. However, it should be stressed that FCs were available in only five obese subjects. So, power issues may be important. Larger cohorts are necessary to study this association in the future. 
In healthy females, AFC and AMH correlate very well (39), but this study shows that this correlation is weak among CCS. This may be due to the fact that follicular AMH expression is lower in CCS treated with gonadotoxic therapies. To our knowledge, no large studies have been performed in female CCS in which the correlation has been studied. Therefore, we cannot draw any firm conclusions based on our small subset analyses regarding the real correlation between AFC and AMH. We believe that it is important to study this association prospectively in a large nationwide cohort, such as the DCOG LATER-VEVO study (40).

We did not correct our analyses for smoking and OCP use. Smoking is linked to ovarian aging in the general population (41). However, we did not find a significant difference in AMH levels between smokers and nonsmokers. The fact that smoking significantly influences ovarian reserve in the general population but not in female CCS may be caused by the large effect of the gonadotoxic treatment that may overshadow the influence of smoking on ovarian reserve. Whether OCP use affects AMH levels is still a matter of debate. In our study, we did not find an association between OCP and AMH. Therefore, we did not include smoking and OCP use as confounders.

In conclusion, low serum AMH is associated with obesity and high insulin levels, and low FC with high insulin levels in a large cohort of adult female CCS. Furthermore, despite its highly predictive value for AFC in the healthy female population, serum AMH seems to correlate only weakly with AFC in CCS.

\section{Supplementary data}

This is linked to the online version of the paper at http://dx.doi.org/10. 1530/EJE-13-0114.

\section{Declaration of interest}

The authors declare that there is no conflict of interest that could be perceived as prejudicing the impartiality of the research reported.

\section{Funding}

W van Dorp is supported by the Paediatric Oncology Center Society for Research (KOCR), Rotterdam. S J C M M Neggers and K Blijdorp are supported by a grant from the KiKa foundation.

\section{Acknowledgements}

The authors would like to thank Dr Saskia Pluijm for her statistical support and valuable suggestions and Dr Patric Delhanty for his support in English writing.

\section{References}

1 Flegal KM, Carroll MD, Ogden CL \& Curtin LR. Prevalence and trends in obesity among US adults, 1999-2008. Journal of the American Medical Association 2010303 235-241. (doi:10.1001/ jama.2009.2014)
2 Guh DP, Zhang W, Bansback N, Amarsi Z, Birmingham CL \& Anis AH. The incidence of co-morbidities related to obesity and overweight: a systematic review and meta-analysis. BMC Public Health 20099 88. (doi:10.1186/1471-2458-9-88)

3 Metwally M, Ong KJ, Ledger WL \& Li TC. Does high body mass index increase the risk of miscarriage after spontaneous and assisted conception? A meta-analysis of the evidence. Fertility and Sterility 200890 714-726. (doi:10.1016/j.fertnstert.2007. 07.1290)

4 Poirier P, Giles TD, Bray GA, Hong Y, Stern JS, Pi-Sunyer FX \& Eckel RH. Obesity and cardiovascular disease: pathophysiology, evaluation, and effect of weight loss: an update of the 1997 American Heart Association Scientific Statement on Obesity and Heart Disease from the Obesity Committee of the Council on Nutrition, Physical Activity, and Metabolism. Circulation 2006 113 898-918. (doi:10.1161/CIRCULATIONAHA.106.171016)

5 van der Steeg JW, Steures P, Eijkemans MJ, Habbema JD, Hompes PG, Burggraaff JM, Oosterhuis GJ, Bossuyt PM, van der Veen F \& Mol BW. Obesity affects spontaneous pregnancy chances in subfertile, ovulatory women. Human Reproduction 200823 324-328. (doi:10.1093/humrep/dem371)

6 Oeffinger KC, Mertens AC, Sklar CA, Yasui Y, Fears T, Stovall M, Vik TA, Inskip PD \& Robison LL. Obesity in adult survivors of childhood acute lymphoblastic leukemia: a report from the Childhood Cancer Survivor Study. Journal of Clinical Oncology 200321 1359-1365. (doi:10.1200/JCO.2003.06.131)

7 Van Dongen-Melman JE, Hokken-Koelega AC, Hahlen K, De Groot A, Tromp CG \& Egeler RM. Obesity after successful treatment of acute lymphoblastic leukemia in childhood. Pediatric Research 199538 86-90. (doi:10.1203/00006450-199507000-00015)

8 Garmey EG, Liu Q, Sklar CA, Meacham LR, Mertens AC, Stovall MA, Yasui Y, Robison LL \& Oeffinger KC. Longitudinal changes in obesity and body mass index among adult survivors of childhood acute lymphoblastic leukemia: a report from the Childhood Cancer Survivor Study. Journal of Clinical Oncology 2008 26 4639-4645. (doi:10.1200/JC0.2008.16.3527)

9 Lie Fong S, Laven JS, Hakvoort-Cammel FG, Schipper I, Visser JA, Themmen AP, de Jong FH \& van den Heuvel-Eibrink MM. Assessment of ovarian reserve in adult childhood cancer survivors using anti-Müllerian hormone. Human Reproduction 200924 982-990. (doi:10.1093/humrep/den487)

10 de Vet A, Laven JS, de Jong FH, Themmen AP \& Fauser BC. AntiMüllerian hormone serum levels: a putative marker for ovarian aging. Fertility and Sterility 200277 357-362. (doi:10.1016/ S0015-0282(01)02993-4)

11 Visser JA, de Jong FH, Laven JS \& Themmen AP. Anti-Müllerian hormone: a new marker for ovarian function. Reproduction 2006 131 1-9. (doi:10.1530/rep.1.00529)

12 Hehenkamp WJ, Looman CW, Themmen AP, de Jong FH, Te Velde ER \& Broekmans FJ. Anti-Müllerian hormone levels in the spontaneous menstrual cycle do not show substantial fluctuation. Journal of Clinical Endocrinology and Metabolism 2006 91 4057-4063. (doi:10.1210/jc.2006-0331)

13 Streuli I, Fraisse T, Pillet C, Ibecheole V, Bischof P \& de Ziegler D. Serum anti-Müllerian hormone levels remain stable throughout the menstrual cycle and after oral or vaginal administration of synthetic sex steroids. Fertility and Sterility 200890 395-400. (doi:10.1016/j.fertnstert.2007.06.023)

14 Tsepelidis S, Devreker F, Demeestere I, Flahaut A, Gervy C \& Englert Y. Stable serum levels of anti-Müllerian hormone during the menstrual cycle: a prospective study in normo-ovulatory women. Human Reproduction 200722 1837-1840. (doi:10.1093/ humrep/dem101)

15 Freeman EW, Gracia CR, Sammel MD, Lin H, Lim LC \& Strauss JF III. Association of anti-Müllerian hormone levels with obesity in late reproductive-age women. Fertility and Sterility $2007 \mathbf{8 7}$ 101-106. (doi:10.1016/j.fertnstert.2006.05.074)

16 Park HT, Cho GJ, Ahn KH, Shin JH, Kim YT, Hur JY, Kim SH, Lee KW \& Kim T. Association of insulin resistance with 
anti-Müllerian hormone levels in women without polycystic ovary syndrome (PCOS). Clinical Endocrinology 201072 26-31. (doi:10. 1111/j.1365-2265.2009.03614.x)

17 Cortet-Rudelli C, Pigny P, Decanter C, Leroy M, MaunouryLefebvre C, Thomas-Desrousseaux P \& Dewailly D. Obesity and serum luteinizing hormone level have an independent and opposite effect on the serum inhibin B level in patients with polycystic ovary syndrome. Fertility and Sterility $2002 \mathbf{7 7}$ 281-287. (doi:10.1016/S0015-0282(01)02968-5)

18 Skalba P, Cygal A, Madej P, Dabkowska-Huc A, Sikora J, Martirosian G, Romanik M \& Olszanecka-Glinianowicz M. Is the plasma anti-Müllerian hormone (AMH) level associated with body weight and metabolic, and hormonal disturbances in women with and without polycystic ovary syndrome? European Journal of Obstetrics \& Gynecology and Reproductive Biology 2011 158 254-259. (doi:10.1016/j.ejogrb.2011.06.006)

19 Dewailly D, Gronier H, Poncelet E, Robin G, Leroy M, Pigny P, Duhamel A \& Catteau-Jonard S. Diagnosis of polycystic ovary syndrome (PCOS): revisiting the threshold values of follicle count on ultrasound and of the serum AMH level for the definition of polycystic ovaries. Human Reproduction 201126 3123-3129. (doi:10.1093/humrep/der297)

20 Kevenaar ME, Meerasahib MF, Kramer P, van de Lang-Born BM, de Jong FH, Groome NP, Themmen AP \& Visser JA. Serum antiMüllerian hormone levels reflect the size of the primordial follicle pool in mice. Endocrinology 2006147 3228-3234. (doi:10.1210/ en.2005-1588)

21 Revised 2003 consensus on diagnostic criteria and long-term health risks related to polycystic ovary syndrome. Fertility and Sterility 200481 19-25.

22 Balen AH, Laven JS, Tan SL \& Dewailly D. Ultrasound assessment of the polycystic ovary: international consensus definitions. Human Reproduction Update 20039 505-514. (doi:10.1093/ humupd/dmg044)

23 Health BNIo. National Institutes of Health (NIH), National Heart, Lung, and Blood Institute (NHLBI). The practical guide: identification, evaluation, and treatment of overweight and obesity in adults. NIH publication OO-4084. 2000.

24 Dobbelsteyn CJ, Joffres MR, MacLean DR \& Flowerdew G. A comparative evaluation of waist circumference, waist-to-hip ratio and body mass index as indicators of cardiovascular risk factors. The Canadian Heart Health Surveys. International Journal of Obesity and Related Metabolic Disorders 200125 652-661. (doi:10.1038/s.ijo.0801582)

25 Wallace TM, Levy JC \& Matthews DR. Use and abuse of HOMA modeling. Diabetes Care 200427 1487-1495. (doi:10.2337/ diacare.27.6.1487)

26 Pimenta N, Santa-Clara H \& Fragoso IJ. Comparison of body composition and body fat distribution of patients following a cardiac rehabilitation program and sedentary patients. Revista Portuguesa de Cardiologia 201029 1163-1180.

27 Green DM, Kawashima T, Stovall M, Leisenring W, Sklar CA, Mertens AC, Donaldson SS, Byrne J \& Robison LL. Fertility of male survivors of childhood cancer: a report from the Childhood Cancer Survivor Study. Journal of Clinical Oncology 201028 332-339. (doi:10.1200/JCO.2009.24.9037)

28 Tucker MA, Meadows AT, Boice JD Jr, Stovall M, Oberlin O, Stone BJ, Birch J, Voute PA, Hoover RN \& Fraumeni JF Jr. Leukemia after therapy with alkylating agents for childhood cancer. Journal of the National Cancer Institute $1987 \mathbf{7 8} 459-464$.

29 Pagano L, De Rosa G, Voso MT, Marra R, Testa A \& Leone G. Prevalence of obesity in young adults with acute lymphoblastic leukemia. International Journal of Clinical and Laboratory Research 199424 117-119. (doi:10.1007/BF02593912)
30 Blijdorp K, van den Heuvel-Eibrink M, Pieters R, Boot A, Sluimer J, van der Lelij AJ \& Neggers S. The limited screening value of insulin-like growth factor-I as a marker for alterations in body composition in very long-term adult survivors of childhood cancer. Pediatric Blood \& Cancer 201159 711-716. (doi:10.1002/ pbc. 24015)

31 Gradmark AM, Rydh A, Renstrom F, De Lucia-Rolfe E, Sleigh A, Nordstrom P, Brage S \& Franks PW. Computed tomography-based validation of abdominal adiposity measurements from ultrasonography, dual-energy X-ray absorptiometry and anthropometry. British Journal of Nutrition $2010 \mathbf{1 0 4} 582-588$. (doi:10.1017/ S0007114510000796)

32 Buyuk E, Seifer DB, Illions E, Grazi RV \& Lieman H. Elevated body mass index is associated with lower serum anti-Müllerian hormone levels in infertile women with diminished ovarian reserve but not with normal ovarian reserve. Fertility and Sterility 201195 2364-2368. (doi:10.1016/j.fertnstert.2011.03.081)

33 Santollo J \& Eckel LA. Estradiol decreases the orexigenic effect of neuropeptide Y, but not agouti-related protein, in ovariectomized rats. Behavioral Brain Research 2008191 173-177. (doi:10.1016/ j.bbr.2008.03.019)

34 Messina MM, Boersma G, Overton JM \& Eckel LA. Estradiol decreases the orexigenic effect of melanin-concentrating hormone in ovariectomized rats. Physiology \& Behavior 2006 88 523-528. (doi:10.1016/j.physbeh.2006.05.002)

35 Clegg DJ, Brown LM, Woods SC \& Benoit SC. Gonadal hormones determine sensitivity to central leptin and insulin. Diabetes 2006 55 978-987. (doi:10.2337/diabetes.55.04.06.db05-1339)

36 Isik S, Ozcan HN, Ozuguz U, Tutuncu YA, Berker D, Alimli AG, Akbaba G, Karademir MA \& Guler S. Evaluation of ovarian reserve based on hormonal parameters, ovarian volume, and antral follicle count in women with type 2 diabetes mellitus. Journal of Clinical Endocrinology and Metabolism 201297 261-269. (doi:10.1210/jc.2011-1923)

37 Kjaer K, Hagen C, Sando SH \& Eshoj O. Epidemiology of menarche and menstrual disturbances in an unselected group of women with insulin-dependent diabetes mellitus compared to controls. Journal of Clinical Endocrinology and Metabolism 199275 524-529. (doi:10.1210/jc.75.2.524)

38 Garris DR \& Garris BL. Diabetes (db/db) mutation-induced ovarian involution: progressive hypercytolipidemia. Experimental Biology and Medicine 2003228 1040-1050.

39 Lie Fong S, Visser JA, Welt CK, Eijkemans MJC, Broekmans FJ, Steegers EAP, Hokken-Koelega ACS, Fauser BCJM, de Jong FH, Schipper I et al. Serum anti-Müllerian hormone levels in healthy females, a nomogram ranging from infancy to adulthood. Journal of Clinical Endocrinology and Metabolism 201297 4650-4655. (doi:10.1210/jc.2012-1440)

40 Overbeek A, van den Berg MH, Kremer LC, van den HeuvelEibrink MM, Tissing WJ, Loonen JJ, Versluys B, Bresters D, Kaspers GJ, Lambalk CB et al. A nationwide study on reproductive function, ovarian reserve, and risk of premature menopause in female survivors of childhood cancer: design and methodological challenges. BMC Cancer 201212 363. (doi:10.1186/1471-240712-363)

41 Plante BJ, Cooper GS, Baird DD \& Steiner AZ. The impact of smoking on anti-Müllerian hormone levels in women aged 38 to 50 years. Menopause 201017 571-576.

Received 4 February 2013

Revised version received 14 March 2013

Accepted 4 April 2013 